\title{
THIRD DOUBLE-LAYER POTENTIAL FOR A GENERALIZED BI-AXIALLY SYMMETRIC HELMHOLTZ EQUATION
}

\author{
T.G. ERGASHEV
}

\begin{abstract}
The double-layer potential plays an important role in solving boundary value problems for elliptic equations, and in studying this potential, the properties of the fundamental solutions of the given equation are used. At present, all fundamental solutions to the generalized bi-axially symmetric Helmholtz equation are known but nevertheless, only for the first of them the potential theory was constructed. In this paper we study the double layer potential corresponding to the third fundamental solution. By using properties of Appell hypergeometric functions of two variables, we prove limiting theorems and derive integral equations involving the density of double-layer potentials in their kernels.
\end{abstract}

Keywords: generalized bi-axially symmetric Helmholtz equation; Green formula; fundamental solution; third double-layer potential; Appell hypergeometric functions of two variables; integral equations with a density of double-layer potential in their kernel.

Mathematics Subject Classification: 35A08, 35J05, 35J15,35J70

\section{INTRODUCTION}

Numerous applications of potential theory can be found in fluid mechanics, elastodynamics, electromagnetics and acoustics. By means of this theory one succeeds to reduce boundary value problems to solving integral equations.

The double layer potential plays an important role in solving boundary value problems for elliptic equations since the separation of variables and the Green's function allows one to obtain explicit solutions only for simplest domains. On one hand, the reducing of boundary value problems by means of double layer potential to integral equations is convenient for theoretical studies on solvability and uniqueness of solutions to boundary value problems. On the other hand, this gives an opportunity for an effective numerical solving of boundary value problems for domains of complicated shapes $[1,2]$.

Applying the methods of complex analysis based on analytic functions, Hilbert [3] first constructed an integral representation for solutions of the following bi-axially symmetric Helmholtz equations

$$
H_{\alpha, \beta}^{\lambda}(u) \equiv u_{x x}+u_{y y}+\frac{2 \alpha}{x} u_{x}+\frac{2 \beta}{y} u_{y}-\lambda^{2} u=0,
$$

where $\alpha, \beta$ and $\lambda$ are constants and $0<2 \alpha, 2 \beta<1$.

Fundamental solutions of equation $\left(H_{\alpha, \beta}^{\lambda}\right)$ were found in work [4]. As $\lambda=0$, all four fundamental solutions $q_{i}\left(x, y ; x_{0}, y_{0}\right), i=\overline{1,4}$ of the equation $H_{\alpha, \beta}^{0}(u)=0$ can be expressed by means

T.G. ERgashev, Third DOUble-LAYER POtential FOR A GENERALized BI-AXIALly Symmetric HELMHOLTZ EQUATION.

(C)Ergashev T.G. 2018.

Submitted August 1, $201 \%$. 
of the Appell hypergeometric function of second kind of two variables $F_{2}\left(a, b_{1}, b_{2} ; c_{1}, c_{2} ; x, y\right)$ defined by the formula $[5,6,7]$

$$
F_{2}\left(a, b_{1}, b_{2} ; c_{1}, c_{2} ; x, y\right)=\sum_{m, n=0}^{\infty} \frac{(a)_{m+n}\left(b_{1}\right)_{m}\left(b_{2}\right)_{n}}{\left(c_{1}\right)_{m}\left(c_{2}\right)_{n} m ! n !} x^{m} y^{n}
$$

where $(a)_{n}$ is the Pochhammer symbol, $(a)_{0}=1,(a)_{n}=a(a+1)(a+2) \ldots(a+n-1)$, $n=1,2, \ldots$

Work [8] belongs to this direction of the studies. In this work there were constructed fundamental solutions of $B$-elliptic equations with lower order terms of form

$$
u_{x x}+u_{y y}+2 \alpha u_{x}+\frac{2 \beta}{y} u_{y}-\lambda^{2} u=0 .
$$

In works [9] and [10], the potential theory was exposed for the simplest degenerating elliptic equation $H_{\alpha, \beta}^{0}(u)=0$ as $\alpha=0$ and $\beta=0$, respectively. In [11] the theory of double layer potential was constructed for equation $\left(H_{\alpha, \beta}^{\lambda}\right)$ as $\lambda=0$ in the domain

$$
\Omega \subset \mathbb{R}_{+}=\{(x, y): x>0, y>0\}
$$

only for the first fundamental solution $q_{1}\left(x, y ; x_{0}, y_{0}\right)$.

In the present work we study the double layer potential corresponding to the third fundamental solution

$$
q_{3}\left(x, y ; x_{0}, y_{0}\right)=k_{3}\left(r^{2}\right)^{-\alpha+\beta-1} y^{1-2 \beta} y_{0}^{1-2 \beta} F_{2}(1+\alpha-\beta ; \alpha, 1-\beta ; 2 \alpha, 2-2 \beta ; \xi, \eta),
$$

where

$$
\begin{aligned}
& k_{3}=\frac{2^{2+2 \alpha-2 \beta}}{4 \pi} \frac{\Gamma(\alpha) \Gamma(1-\beta) \Gamma(1+\alpha-\beta)}{\Gamma(2 \alpha) \Gamma(2-2 \beta)}, \\
& \left.\begin{array}{l}
r^{2} \\
r_{1}^{2} \\
r_{2}^{2}
\end{array}\right\}=\left(\begin{array}{l}
x-x_{0} \\
x+x_{0} \\
x-x_{0}
\end{array}\right)^{2}+\left(\begin{array}{l}
y-y_{0} \\
y-y_{0} \\
y+y_{0}
\end{array}\right)^{2}, \quad \xi=\frac{r^{2}-r_{1}^{2}}{r^{2}}, \quad \eta=\frac{r^{2}-r_{2}^{2}}{r^{2}} .
\end{aligned}
$$

It is straightforward to check that the function $q_{3}\left(x, y ; x_{0}, y_{0}\right)$ possesses the following properties:

$$
\begin{aligned}
& \left.\frac{\partial q_{3}\left(x, y ; x_{0}, y_{0}\right)}{\partial x}\right|_{x=0}=0 \\
& \left.q_{3}\left(x, y ; x_{0}, y_{0}\right)\right|_{y=0}=0 .
\end{aligned}
$$

\section{GREEN'S FORMULA}

We consider the identity

$$
x^{2 \alpha} y^{2 \beta}\left[u H_{\alpha, \beta}^{0}(v)-v H_{\alpha, \beta}^{0}(u)\right]=\frac{\partial}{\partial x}\left[x^{2 \alpha} y^{2 \beta}\left(v_{x} u-v u_{x}\right)\right]+\frac{\partial}{\partial y}\left[x^{2 \alpha} y^{2 \beta}\left(v_{y} u-v u_{y}\right)\right] .
$$

Integrating both sides of this identity over a domain $\Omega$ located in the first quarter $(x>0, y>0)$ and employing the Ostrogradsky formula, we get

$$
\iint_{\Omega} x^{2 \alpha} y^{2 \beta}\left[u H_{\alpha, \beta}^{0}(v)-v H_{\alpha, \beta}^{0}(u)\right] d x d y=\int_{S} x^{2 \alpha} y^{2 \beta} u\left(v_{x} d y-v_{y} d x\right)-x^{2 \alpha} y^{2 \beta} v\left(u_{x} d y-u_{y} d x\right),
$$

where $S=\partial \Omega$ is the boundary of the domain $\Omega$.

Green's formula (2.1) is obtained under the following assumptions: the functions $u(x, y)$, $v(x, y)$ and its first partial derivatives are continuous in the closed domain $\bar{\Omega}$, second order partial derivatives are continuous inside $\Omega$ and the integrals over $\Omega$ involving $H_{\alpha, \beta}^{0}(u)$ and 
$H_{\alpha, \beta}^{0}(v)$ are well-defined. If $H_{\alpha, \beta}^{0}(u)$ and $H_{\alpha, \beta}^{0}(v)$ are not continuous up to $S$, then these are improper integrals obtained as limits over each sequence of domains $\Omega_{n}$ located inside $\Omega$ as they tend to $\Omega$ so that each internal point of $\Omega$ is also internal for domains $\Omega_{n}$ starting with some index $n$.

If $u(x, y)$ and $v(x, y)$ solve the equation $H_{\alpha, \beta}^{0}(u)=0$, by formula (2.1) we have

$$
\int_{S} x^{2 \alpha} y^{2 \beta}\left(u \frac{\partial v}{\partial n}-v \frac{\partial u}{\partial n}\right) d s=0 .
$$

Here

$$
\frac{\partial}{\partial n}=\frac{d y}{\partial s} \frac{\partial}{\partial x}-\frac{d x}{\partial s} \frac{\partial}{\partial y}
$$

is the operator of differentiation along the outward normal $n$ to the curve $S$ and

$$
\frac{d y}{d s}=\cos (n, x), \quad \frac{d x}{d s}=-\cos (n, y)
$$

are direction cosines for this normal.

Letting $v \equiv 1$ in formula $(2.1)$ and replacing $u$ by $u^{2}$, we obtain

$$
\iint_{\Omega} x^{2 \alpha} y^{2 \beta}\left[u_{x}^{2}+u_{y}^{2}\right] d x d y=\int_{S} x^{2 \alpha} y^{2 \beta} u \frac{\partial u}{\partial n} d s,
$$

where $u(x, y)$ is a solution of the equation $H_{\alpha, \beta}^{0}(u)=0$. Letting $v \equiv 1$ in formula (2.2), we obtain

$$
\int_{S} x^{2 \alpha} y^{2 \beta} \frac{\partial u}{\partial n} d s=0
$$

that is, the integral of the normal derivative of a solution to the equation $H_{\alpha, \beta}^{0}(u)=0$ with the weight $x^{2 \alpha} y^{2 \beta}$ over the boundary of the domain vanishes.

\section{Double LAYer Potential $w^{(3)}\left(x_{0}, y_{0}\right)$}

Let $\Omega$ be a domain enveloped by the segments $(0, a)$ and $(0, b)$ in the axes $x$ and $y$, respectively, and by a curve $\Gamma$ with end-points $A(a, 0)$ and $B(0, b)$ located in the first quarter $x>0, y>0$ in the plane $\mathbb{R}^{2}$. A parametric equation for the curve $\Gamma$ is $x=x(s)$ and $y=y(s), s \in[0, l]$, where $s$ is the arc length measured from the point $B$. For the curve $\Gamma$ we assume that

1 ) the functions $x=x(s)$ and $y=y(s)$ possess continuous derivatives $x^{\prime}(s)$ and $y^{\prime}(s)$ on the segment $[0, l]$ not vanishing simultaneously; the second derivatives $x^{\prime \prime}(s)$ and $y^{\prime \prime}(s)$ satisfy the Hölder condition with an exponent $\varepsilon, 0<\varepsilon<1$, on $[0, l]$, where $l$ is the length of the curve $\Gamma$;

2 ) in the vicinities of the points $A(a, 0)$ and $B(0, b)$ on the curve $\Gamma$ the conditions hold:

$$
\left|\frac{d x}{d s}\right| \leqslant C y^{1+\varepsilon}(s), \quad\left|\frac{d y}{d s}\right| \leqslant C x^{1+\varepsilon}(s),
$$

where $C=$ const. The coordinates of points in the curve $\Gamma$ are denoted by $(x, y)$.

We consider the integral

$$
w^{(3)}\left(x_{0}, y_{0}\right)=\int_{0}^{l} x^{2 \alpha} y^{2 \beta} \mu_{3}(s) \frac{\partial q_{3}\left(x, y ; x_{0}, y_{0}\right)}{\partial n} d s,
$$

where $\mu_{3}(s)$ is a continuous function in the segment $[0, l]$, and $q_{3}\left(x, y ; x_{0}, y_{0}\right)$ is a fundamental solution of the equation $H_{\alpha, \beta}^{0}(u)=0$ defined by formula (1.1).

Integral (3.2) is called third double layer potential with a density $\mu_{3}(s)$. It is obvious that $w^{(3)}\left(x_{0}, y_{0}\right)$ is a regular solution to the equation $H_{\alpha, \beta}^{0}(u)=0$ in each domain located in the 
first quarter having common points neither with $\Gamma$ no with the axes $x$ and $y$. As in the case of logarithmic potential, one can show the existence of the double layer potential (3.2) at the points of the curve $\Gamma$ for a bounded density $\mu_{3}(s)$.

Lemma 1. The following formulae hold:

$$
w^{(3)}\left(x_{0}, y_{0}\right)=\left\{\begin{array}{cll}
j\left(x_{0}, y_{0}\right)-1 & \text { if } \quad\left(x_{0}, y_{0}\right) \in \Omega, \\
j\left(x_{0}, y_{0}\right)-\frac{1}{2} & \text { if } \quad\left(x_{0}, y_{0}\right) \in \Gamma, \\
j\left(x_{0}, y_{0}\right) & \text { if } \quad\left(x_{0}, y_{0}\right) \notin \bar{\Omega}
\end{array}\right.
$$

where $\bar{\Omega}:=\Omega \cup \Gamma$;

$$
\begin{aligned}
j\left(x_{0}, y_{0}\right)=(1-2 \beta) k_{3} y_{0}^{1-2 \beta} \int_{0}^{a} & x^{2 \alpha}\left(\left(x-x_{0}\right)^{2}+y_{0}^{2}\right)^{-\alpha+\beta-1} \\
& F\left(1+\alpha-\beta, \alpha ; 2 \alpha ; \frac{-4 x x_{0}}{\left(x-x_{0}\right)^{2}+y_{0}^{2}}\right) d x .
\end{aligned}
$$

Here

$$
F(a, b ; c ; z)=\sum_{k=0}^{\infty} \frac{(a)_{k}(b)_{k}}{(c)_{k} k !} z^{k}
$$

is the Gauss hypergeometric function.

Proof. Case 1. Assume that the point $\left(x_{0}, y_{0}\right)$ is located inside $\Omega$. We cut out a circle of a small radius $\rho$ centered at $\left(x_{0}, y_{0}\right)$ from the domain $\Omega$ and by $\Omega_{\rho}$ we denote the remaining part of the domain $\Omega$, while $C_{\rho}$ stands for the circumference of the cut circle. In the domain $\Omega_{\rho}$ the function $q_{3}\left(x, y ; x_{0}, y_{0}\right)$ is a regular solution of the equation $H_{\alpha, \beta}^{0}(u)=0$. Employing the formula for the derivative of the Appell hypergeometric function [12]

$\frac{\partial^{m+n} F_{2}\left(a ; b_{1}, b_{2} ; c_{1}, c_{2} ; x, y\right)}{\partial x^{m} \partial y^{n}}=\frac{(a)_{m+n}\left(b_{1}\right)_{m}\left(b_{2}\right)_{n}}{\left(c_{1}\right)_{m}\left(c_{2}\right)_{n}} F_{2}\left(a+m+n ; b_{1}+m, b_{2}+n ; c_{1}+m, c_{2}+n ; x, y\right)$,

we obtain

$$
\frac{\partial q_{3}\left(x, y ; x_{0}, y_{0}\right)}{\partial x}=-2(1+\alpha-\beta) k_{3}\left(r^{2}\right)^{-\alpha+\beta-2} y^{1-2 \beta} y_{0}^{1-2 \beta} P\left(x, y ; x_{0}, y_{0}\right),
$$

where

$$
\begin{aligned}
P\left(x, y ; x_{0}, y_{0}\right) & =\left(x-x_{0}\right) F_{2}(1+\alpha-\beta ; \alpha, 1-\beta ; 2 \alpha, 2-2 \beta ; \xi, \eta) \\
& +x_{0} F_{2}(2+\alpha-\beta ; 1+\alpha, 1-\beta ; 1+2 \alpha, 2-2 \beta ; \xi, \eta) \\
& +\left(x-x_{0}\right)\left[\frac{(1+\alpha-\beta) \alpha}{2 \alpha} \xi F_{2}(2+\alpha-\beta ; 1+\alpha, 1-\beta ; 1+2 \alpha, 2-2 \beta ; \xi, \eta)\right. \\
& \left.+\frac{1-\beta}{2-2 \beta} \eta F_{2}(2+\alpha-\beta ; \alpha, 2-\beta ; 2 \alpha, 3-2 \beta ; \xi, \eta)\right] .
\end{aligned}
$$

Applying then a known relation [5]

$$
\begin{array}{r}
\frac{b_{1}}{c_{1}} x F_{2}\left(a+1 ; b_{1}+1, b_{2} ; c_{1}+1, c_{2} ; x, y\right)+\frac{b_{2}}{c_{2}} y F_{2}\left(a+1 ; b_{1}, b_{2}+1 ; c_{1}, c_{2}+1 ; x, y\right) \\
=F_{2}\left(a+1 ; b_{1}, b_{2} ; c_{1}, c_{2} ; x, y\right)-F_{2}\left(a ; b_{1}, b_{2} ; c_{1}, c_{2} ; x, y\right)
\end{array}
$$


to the square brackets in (3.7), we get:

$$
\begin{aligned}
\frac{\partial q_{3}\left(x, y ; x_{0}, y_{0}\right)}{\partial x}= & -2(1+\alpha-\beta) k_{3}\left(r^{2}\right)^{-\alpha+\beta-2} y^{1-2 \beta} y_{0}^{1-2 \beta} \\
& \cdot\left[x_{0} F_{2}(2+\alpha-\beta ; 1+\alpha, 1-\beta ; 1+2 \alpha, 2-2 \beta ; \xi, \eta)\right. \\
& \left.+\left(x-x_{0}\right) F_{2}(2+\alpha-\beta ; \alpha, 1-\beta ; 2 \alpha, 2-2 \beta ; \xi, \eta)\right] .
\end{aligned}
$$

In the same way we find:

$$
\begin{aligned}
\frac{\partial q_{3}\left(x, y ; z_{0}, y_{0}\right)}{\partial y}= & -2(1+\alpha-\beta) k_{3}\left(r^{2}\right)^{-\alpha+\beta-2} y^{1-2 \beta} y_{0}^{1-2 \beta} \\
& \cdot\left[y_{0} F_{2}(2+\alpha-\beta ; \alpha, 2-\beta ; 2 \alpha, 3-2 \beta ; \xi, \eta)\right. \\
& \left.+\left(y-y_{0}\right) F_{2}(2+\alpha-\beta ; \alpha, 1-\beta ; 2 \alpha, 2-2 \beta ; \xi, \eta)\right] \\
& +(1-2 \beta) k_{3}\left(r^{2}\right)^{-\alpha+\beta-1} y^{-2 \beta} y_{0}^{1-2 \beta} \\
& \cdot F_{2}(1+\alpha-\beta ; \alpha, 1-\beta ; 2 \alpha, 2-2 \beta ; \xi, \eta) .
\end{aligned}
$$

Employing (3.8) and (3.9), by (1.1), (2.3) and (2.4) we find:

$$
\frac{\partial q_{3}\left(x, y ; x_{0}, y_{0}\right)}{\partial n}=(1+\alpha-\beta) k_{3}\left(r^{2}\right)^{-\alpha+\beta-2} y^{-2 \beta} y_{0}^{1-2 \beta} Q\left(x, y ; x_{0}, y_{0}\right)
$$

where

$$
\begin{aligned}
Q\left(x, y ; x_{0}, y_{0}\right)= & -r^{2} y F_{2}(2+\alpha-\beta ; \alpha, 1-\beta ; 2 \alpha, 2-2 \beta ; \xi, \eta) \frac{\partial}{\partial n}\left[\ln r^{2}\right] \\
& -2 y y_{0} F_{2}(2+\alpha-\beta ; 1+\alpha, 1-\beta ; 1+2 \alpha, 2-2 \beta ; \xi, \eta) \frac{d x}{d s} \\
& +2 x_{0} y F_{2}(2+\alpha-\beta ; \alpha, 2-\beta ; 2 \alpha, 3-2 \beta ; \xi, \eta) \frac{d y}{d s} \\
& +(1-2 \beta) r^{2} F_{2}(1+\alpha-\beta ; \alpha, 1-\beta ; 2 \alpha, 2-2 \beta ; \xi, \eta) \frac{d x}{d s}
\end{aligned}
$$

We integrate the normal derivative $\frac{\partial}{\partial n} q_{3}\left(x, y ; x_{0}, y_{0}\right)$ with the weight $x^{2 \alpha} y^{2 \beta}$ over the boundary of the domain $\Omega_{\rho}$ and by (2.5) we get

$$
\begin{aligned}
& \left.\int_{0}^{a} x^{2 \alpha}\left[y^{2 \beta} \frac{\partial q_{3}\left(x, y ; x_{0}, y_{0}\right)}{\partial n}\right]\right|_{y=0} d x+\int_{0}^{l} x^{2 \alpha} y^{2 \beta} \mu_{3}(s) \frac{\partial q_{3}\left(x, y ; x_{0}, y_{0}\right)}{\partial n} d s \\
& -\lim _{\rho \rightarrow 0} \int_{C_{\rho}} x^{2 \alpha} y^{2 \beta} \frac{\partial q_{3}\left(x, y ; x_{0}, y_{0}\right)}{\partial n} d s-\left.\int_{0}^{b} x^{2 \alpha} y^{2 \beta} \frac{\partial q_{3}\left(x, y ; x_{0}, y_{0}\right)}{\partial n}\right|_{x=0} d y=0 .
\end{aligned}
$$

Then in view of (3.2) and (1.4) we have

$$
w_{1}^{(3)}\left(x_{0}, y_{0}\right)=\lim _{\rho \rightarrow 0} \int_{C_{\rho}} x^{2 \alpha} y^{2 \beta} \frac{\partial q_{3}\left(x, y ; x_{0}, y_{0}\right)}{\partial n} d s+\left.\int_{0}^{a} x^{2 \alpha}\left[y^{2 \beta} \frac{\partial q_{3}\left(x, y ; x_{0}, y_{0}\right)}{\partial y}\right]\right|_{y=0} d x \text {. }
$$

Substituting (3.10) into (3.11), we find:

$$
w_{1}^{(3)}\left(x_{0}, y_{0}\right)=k_{3} y_{0}^{1-2 \beta} \lim _{\rho \rightarrow 0}\left\{(1+\alpha-\beta)\left[-J_{1}-2 y_{0} J_{2}+2 x_{0} J_{3}\right]+J_{4}\right\}+J_{5},
$$

where

$$
J_{1}=\int_{C_{\rho}} x^{2 \alpha} y\left(r^{2}\right)^{-\alpha+\beta-1} F_{2}(2+\alpha-\beta ; \alpha, 1-\beta ; 2 \alpha, 2-2 \beta ; \xi, \eta) \frac{\partial}{\partial n}\left[\ln r^{2}\right] d s,
$$




$$
\begin{aligned}
& J_{2}=\int_{C_{\rho}} x^{2 \alpha} y\left(r^{2}\right)^{-\alpha+\beta-2} F_{2}(2+\alpha-\beta ; 1+\alpha, 1-\beta ; 1+2 \alpha, 2-2 \beta ; \xi, \eta) \frac{d x(s)}{d s} d s, \\
& J_{3}=\int_{C_{\rho}} x^{2 \alpha} y\left(r^{2}\right)^{-\alpha+\beta-2} F_{2}(2+\alpha-\beta ; \alpha, 2-\beta ; 2 \alpha, 3-2 \beta ; \xi, \eta) \frac{d y(s)}{d s} d s, \\
& J_{4}=(1-2 \beta) \int_{C_{\rho}} x^{2 \alpha}\left(r^{2}\right)^{-\alpha+\beta-1} F_{2}(1+\alpha-\beta ; \alpha, 1-\beta ; 2 \alpha, 2-2 \beta ; \xi, \eta) \frac{d x(s)}{d s} d s, \\
& J_{5}=\left.\int_{0}^{a} x^{2 \alpha}\left[y^{2 \beta} \frac{\partial q_{3}\left(x, y ; x_{0}, y_{0}\right)}{\partial y}\right]\right|_{y=0} d x .
\end{aligned}
$$

Passing to the polar coordinates

$$
x=x_{0}+\rho \cos \varphi, \quad y=y_{0}+\rho \sin \varphi
$$

in the integral $J_{1}$, we obtain

$$
J_{1}=\int_{0}^{2 \pi}\left(x_{0}+\rho \cos \varphi\right)^{2 \alpha}\left(y_{0}+\rho \sin \varphi\right)\left(\rho^{2}\right)^{-\alpha+\beta-1} F_{2}(2+\alpha-\beta ; \alpha, 1-\beta ; 2 \alpha, 2-2 \beta ; \xi, \eta) d \varphi .
$$

Let us study the integrand in (3.14). Applying successively the known formulae [13]

$F_{2}\left(a ; b_{1}, b_{2} ; c_{1}, c_{2} ; x, y\right)=\sum_{i=0}^{\infty} \frac{(a)_{i}\left(b_{1}\right)_{i}\left(b_{2}\right)_{i}}{\left(c_{1}\right)_{i}\left(c_{2}\right)_{i} i !} x^{i} y^{i} F\left(a+i, b_{1}+i ; c_{1}+i ; x\right) F\left(a+i, b_{2}+i ; c_{2}+i ; y\right)$ and

we obtain

$$
F(a, b ; c, x)=(1-x)^{-b} F\left(c-a, b ; c, \frac{x}{x-1}\right)
$$

$$
\begin{aligned}
F_{2}\left(a ; b_{1}, b_{2} ; c_{1}, c_{2} ; x, y\right)= & \frac{(1-x)^{-b_{1}}}{(1-y)^{b_{2}}} \sum_{i=0}^{\infty} \frac{(a)_{i}\left(b_{1}\right)_{i}\left(b_{2}\right)_{i}}{\left(c_{1}\right)_{i}\left(c_{2}\right)_{i} i !}\left(\frac{x}{1-x}\right)^{i}\left(\frac{y}{1-y}\right)^{i} \\
& \cdot F\left(c_{1}-a, b_{1}+i ; c_{1}+i ; \frac{x}{x-1}\right) \\
& \cdot F\left(c_{2}-a, b_{2}+i ; c_{2}+i ; \frac{y}{y-1}\right) .
\end{aligned}
$$

Employing now formula (3.16), we write out the hypergeometric Appell function $F_{2}(2+\alpha-\beta ; \alpha, 1-\beta ; 2 \alpha, 2-2 \beta ; \xi, \eta)$ as

$$
\begin{aligned}
F_{2}(2+\alpha-\beta ; \alpha, 1-\beta ; 2 \alpha, 2-2 \beta ; \xi, \eta)= & \left(\rho^{2}\right)^{1+\alpha-\beta}\left(\rho^{2}+4 x_{0}^{2}+4 x_{0} \rho \cos \varphi\right)^{-\alpha} \\
& \cdot\left(\rho^{2}+4 y_{0}^{2}+4 y_{0} \rho \sin \varphi\right)^{\beta-1} P_{11},
\end{aligned}
$$

where

$$
\begin{aligned}
P_{11}=\sum_{i=0}^{\infty} & \frac{(2+\alpha-\beta)_{i}(\alpha)_{i}(1-\beta)_{i}}{(2 \alpha)_{i}(2-2 \beta)_{i} i !}\left(\frac{4 x_{0}^{2}+4 x_{0} \rho \cos \varphi}{\rho^{2}+4 x_{0}^{2}+4 x_{0} \rho \cos \varphi}\right)^{i}\left(\frac{4 y_{0}^{2}+4 y_{0} \rho \sin \varphi}{\rho^{2}+4 y_{0}^{2}+4 y_{0} \rho \sin \varphi}\right)^{i} \\
& \cdot F\left(\alpha+\beta-2, \alpha+i ; 2 \alpha+i ; \frac{4 x_{0}^{2}+4 x_{0} \rho \cos \varphi}{\rho^{2}+4 x_{0}^{2}+4 x_{0} \rho \cos \varphi}\right) \\
& \cdot F\left(-\alpha-\beta, 1-\beta+i ; 2-2 \beta+i ; \frac{4 y_{0}^{2}+4 y_{0} \rho \sin \varphi}{\rho^{2}+4 y_{0}^{2}+4 y_{0} \rho \sin \varphi}\right) .
\end{aligned}
$$


Employing the known formula for $F(a, b ; c ; 1)[14]$

$$
F(a, b ; c ; 1)=\frac{\Gamma(c) \Gamma(c-a-b)}{\Gamma(c-a) \Gamma(c-b)}, c \neq 0,-1,-2, \ldots, \operatorname{Re}(c-a-b)>0,
$$

we obtain

$$
\lim _{\rho \rightarrow 0} P_{11}=\frac{\Gamma(2 \alpha) \Gamma(2-2 \beta)}{\Gamma(2+\alpha-\beta) \Gamma(1-\beta) \Gamma(\alpha)} .
$$

Thus, by (3.14), (3.17) and (3.19), we finally get

$$
-(1+\alpha-\beta) k_{30}^{1-2 \beta} \lim _{\rho \rightarrow 0} J_{1}=-1 .
$$

Since

$$
\lim _{\rho \rightarrow 0} \rho \ln \rho=0
$$

we have

$$
\lim _{\rho \rightarrow 0} J_{2}=\lim _{\rho \rightarrow 0} J_{3}=\lim _{\rho \rightarrow 0} J_{4}=0 .
$$

Finally, we proceed to the integral $J_{5}$, which in accordance with formula (3.9) can be reduced to (3.4), that is,

$$
J_{5}=j\left(x_{0}, y_{0}\right) .
$$

Now, by (3.20)-(3.23), it follows from (3.12) that at the point $\left(x_{0}, y_{0}\right) \in \Omega$, the identity holds:

$$
w_{1}^{(3)}\left(x_{0}, y_{0}\right)=j\left(x_{0}, y_{0}\right)-1 .
$$

Case 2. Assume that point $\left(x_{0}, y_{0}\right)$ coincides with some point $M_{0}$ on the curve $\Gamma$. We take a circumference of a small radius $\rho$ centered at the point $\left(x_{0}, y_{0}\right)$. This circumference cut out a part $\Gamma_{\rho}$ of the curve $\Gamma$. The remaining part of the curve is denoted by $\Gamma-\Gamma_{\rho}$. We denote by $C_{\rho}^{\prime}$ a part of the circumference $C_{\rho}$ located inside the domain $\Omega$ and we consider the domain $\Omega_{\rho}$ enveloped by the curves $\Gamma-\Gamma_{\rho}, C_{\rho}^{\prime}$ and by the segments $[0, a]$ and $[0, b]$ on the axes $x$ and $y$, respectively. Then we have

$$
w_{1}^{(3)}\left(x_{0}, y_{0}\right) \equiv \int_{0}^{l} x^{2 \alpha} y^{2 \beta} \frac{\partial q_{3}\left(x, y ; x_{0}, y_{0}\right)}{\partial n} d s=\lim _{\rho \rightarrow 0} \int_{\Gamma-\Gamma_{\rho}} x^{2 \alpha} y^{2 \beta} \frac{\partial q_{3}\left(x, y ; x_{0}, y_{0}\right)}{\partial n} d s .
$$

Since the point $\left(x_{0}, y_{0}\right)$ lies outside this domain, the function $q_{3}\left(x, y ; x_{0}, y_{0}\right)$ is a regular solution of the equation $H_{\alpha, \beta}^{0}(u)=0$ in this domain and by (2.5), the identity holds:

$$
\begin{aligned}
\int_{\Gamma-\Gamma_{\rho}} x^{2 \alpha} y^{2 \beta} \frac{\partial q_{3}\left(x, y ; x_{0}, y_{0}\right)}{\partial n} d s= & \left.\int_{0}^{a} x^{2 \alpha}\left[y^{2 \beta} \frac{\partial q_{3}\left(x, y ; x_{0}, y_{0}\right)}{\partial y}\right]\right|_{y=0} d x \\
& +\left.\int_{0}^{b} x^{2 \alpha} y^{2 \beta} \frac{\partial q_{3}\left(x, y ; x_{0}, y_{0}\right)}{\partial x}\right|_{x=0} d y \\
& +\int_{C_{\rho}} x^{2 \alpha} y^{2 \beta} \frac{\partial}{\partial n}\left\{q_{3}\left(x, y ; x_{0}, y_{0}\right)\right\} d s
\end{aligned}
$$

Substituting (3.25) into (3.24) and taking into consideration (3.23) and (1.4), we obtain

$$
w_{1}^{(3)}\left(x_{0}, y_{0}\right)=j\left(x_{0}, y_{0}\right)+\lim _{\rho \rightarrow 0} \int_{C_{\rho}} x^{2 \alpha} y^{2 \beta} \frac{\partial q_{3}\left(x, y ; x_{0}, y_{0}\right)}{\partial n} d s .
$$


Introducing once again polar coordinates (3.13) centered at the point $\left(x_{0}, y_{0}\right)$ in the integral

$$
\int_{C_{\rho}} x^{2 \alpha} y^{2 \beta} \frac{\partial}{\partial n}\left\{q_{3}\left(x, y ; x_{0}, y_{0}\right)\right\} d s
$$

and passing to the limit as $\rho \rightarrow 0$, we get

$$
\lim _{\rho \rightarrow 0} \int_{C_{\rho}} x^{2 \alpha} y^{2 \beta} \frac{\partial}{\partial n}\left\{q_{3}\left(x, y ; x_{0}, y_{0}\right)\right\} d s=-\frac{1}{2} .
$$

Thus,

$$
w_{1}^{(3)}\left(x_{0}, y_{0}\right)=j\left(x_{0}, y_{0}\right)-\frac{1}{2} .
$$

Case 3. Finally, suppose that the point $\left(x_{0}, y_{0}\right)$ is located outside the domain $\Omega$. Then $q_{3}\left(x, y ; x_{0}, y_{0}\right)$ is a regular solution to the equation $H_{\alpha, \beta}^{0}(u)=0$ inside the domain $\Omega$ possessing continuous derivatives up to the contour $\Gamma$ and by $(2.5)$

$w_{1}^{(3)}\left(x_{0}, y_{0}\right) \equiv \int_{0}^{l} x^{2 \alpha} y^{2 \beta} \frac{\partial}{\partial n}\left\{q_{3}\left(x, y ; x_{0}, y_{0}\right)\right\} d s=\left.\int_{0}^{a} x^{2 \alpha}\left[y^{2 \beta} \frac{\partial q_{3}\left(x, y ; x_{0}, y_{0}\right)}{\partial y}\right]\right|_{y=0} d x=j\left(x_{0}, y_{0}\right)$.

Lemma 2. The following formulae hold true:

$$
w^{(2)}\left(0, y_{0}\right)= \begin{cases}j\left(0, y_{0}\right)-1 & \text { if } y_{0} \in(0, b) \\ j\left(0, y_{0}\right)-\frac{1}{2} & \text { if } y_{0}=0 \quad \text { or } \quad y_{0}=b \\ j\left(0, y_{0}\right) & \text { if } b<y_{0},\end{cases}
$$

where

$$
j\left(0, y_{0}\right)=\frac{1-2 \beta}{1+2 \alpha}\left(\frac{a^{2}}{y_{0}^{2}+a^{2}}\right)^{\frac{1}{2}+\alpha} k_{3} F\left(\frac{1}{2}+\beta, \frac{1}{2}+\alpha ; \frac{3}{2}+\alpha ; \frac{a^{2}}{y_{0}^{2}+a^{2}}\right) .
$$

Proof. First we study the function $j\left(x_{0}, y_{0}\right)$ defined by formula (3.4) at $x_{0}=0$ :

$$
j\left(0, y_{0}\right)=(1-2 \beta) k_{3} y_{0}^{1-2 \beta} \int_{0}^{a} x^{2 \alpha}\left(x^{2}+y_{0}^{2}\right)^{-\alpha+\beta-1} d x .
$$

Employing the known formula [14]

$$
\int_{0}^{a} x^{\lambda-1}\left(x^{2}+b^{2}\right)^{\nu} d x=\frac{1}{\lambda} b^{2 \nu} a^{\lambda} F\left(-\nu, \frac{\lambda}{2}, \frac{\lambda+2}{2} ; \frac{-a^{2}}{b^{2}}\right), \quad(a b>0, \lambda>0),
$$

we get:

$$
j\left(0, y_{0}\right)=(1-2 \beta) k_{3} a^{1+2 \beta} y_{0}^{-1-2 \beta} F\left(\alpha-\beta+1, \frac{1}{2}+\alpha ; \frac{3}{2}+\alpha ; \frac{-a^{2}}{y_{0}^{2}}\right) .
$$

Using formula (3.15), we get the function $j\left(0, y_{0}\right)$ introduced by formula (3.26). Taking into consideration known formula (3.18) for $F(a, b ; c ; 1)$ and the value $k_{3}$ in formula (1.2), identity (3.26) implies immediately that $j(0,0)=1$.

Suppose that the point $\left(x_{0}, y_{0}\right)$ is located on the axis $y$ and first we assume that $y_{0} \in(0, b)$. We draw the straight line $x=h$ for a sufficiently small $h>0$ and consider the domain $\Omega_{h}$ being a part of the domain $\Omega$ located to the right of the line $x=h$. Applying formula (2.5), we obtain

$$
w_{1}^{(3)}\left(0, y_{0}\right)=J_{6}+J_{7}
$$


where

$$
J_{6}=\left.\lim _{h \rightarrow 0} \int_{h}^{a} x^{2 \alpha} y^{2 \beta} \frac{\partial q_{3}\left(x, y ; 0, y_{0}\right)}{\partial y}\right|_{y=0} d x, \quad J_{7}=\left.\lim _{h \rightarrow 0} \int_{0}^{y_{1}} y^{2 \beta} x^{2 \alpha} \frac{\partial q_{3}\left(x, y ; 0, y_{0}\right)}{\partial x}\right|_{x=h} d x .
$$

Here $y_{1}$ is the ordinate of the intersection of the curve $\Gamma$ with the line $x=h$.

It is easy to observe that

$$
J_{6}=j\left(0, y_{0}\right) .
$$

Now we consider the second term in (3.28), which (3.8) casts into the form

$$
J_{7}=-2(1-\alpha-\beta) k_{3} y_{0}^{1-2 \beta} J_{8},
$$

where

$$
J_{8}=h^{1+2 \alpha} \int_{0}^{y_{1}} y \frac{F\left(2+\alpha-\beta, 1-\beta ; 2-2 \beta ;-\frac{4 y y_{0}}{\left(y-y_{0}\right)^{2}+h^{2}}\right)}{\left[\left(y-y_{0}\right)^{2}+h^{2}\right]^{2+\alpha-\beta}} d y .
$$

We are going to transform $J_{8}$. Employing formula (3.15), we obtain:

$$
J_{8}=h^{1+2 \alpha} \int_{0}^{y_{1}} y \frac{F\left(-\alpha-\beta, 1-\beta ; 2-2 \beta ; \frac{4 y y_{0}}{\left(y+y_{0}\right)^{2}+h^{2}}\right)}{\left[\left(y-y_{0}\right)^{2}+h^{2}\right]^{1+\alpha}\left[\left(y+y_{0}\right)^{2}+h^{2}\right]^{1-\beta}} d x .
$$

We make the change of the variable replacing $y$ by $y=y_{0}+h t$ :

$$
J_{8}\left(h, y_{0}\right)=\int_{l_{1}}^{l_{2}}\left(y_{0}+h t\right) \frac{F\left(-\alpha-\beta, 1-\beta ; 2-2 \beta, \frac{4 y_{0}\left(y_{0}+h t\right)}{\left(2 y_{0}+h t\right)^{2}+h^{2}}\right)}{\left(1+t^{2}\right)^{\alpha+1}\left[\left(2 y_{0}+h t\right)^{2}+h^{2}\right]^{1-\beta}} d t
$$

where

Taking into consideration that

$$
l_{1}=-\frac{y_{0}}{h}, l_{2}=\frac{y_{1}-y_{0}}{h} .
$$

$$
\begin{aligned}
\lim _{h \rightarrow 0} F\left(-\alpha-\beta, 1-\beta ; 2-2 \beta, \frac{4 y_{0}\left(y_{0}+h t\right)}{\left(2 y_{0}+h t\right)^{2}+h^{2}}\right) & =F(-\alpha-\beta, 1-\beta ; 2-2 \beta ; 1) \\
& =\frac{\Gamma(2-2 \beta) \Gamma(1+\alpha)}{\Gamma(2+\alpha-\beta) \Gamma(1-\beta)}
\end{aligned}
$$

and

$$
\int_{-\infty}^{+\infty} \frac{d t}{\left(1+t^{2}\right)^{\alpha+1}}=\frac{\pi \Gamma(2 \alpha)}{2^{2 \alpha-1} \alpha \Gamma^{2}(\alpha)}
$$

by (3.29)-(3.31) we find

$$
w_{1}^{(3)}\left(0, y_{0}\right)=j\left(0, y_{0}\right)-1 .
$$

The other three cases as $y_{0}=0, y_{0}=b$ and $y_{0}>b$ can be treated in the same way as the first case.

Lemma 3. For all points $(x, y)$ and $\left(x_{0}, y_{0}\right) \in R_{+}^{2}$ obeying $x \neq x_{0}$ and $y \neq y_{0}$, the inequality holds:

$$
\begin{aligned}
\left|q_{3}\left(x, y ; x_{0}, y_{0}\right)\right| \leqslant & \frac{\Gamma(\alpha) \Gamma(1-\beta)}{\pi \Gamma(1+\alpha-\beta)} \frac{4^{\alpha-\beta} y^{1-2 \beta} y_{0}^{1-2 \beta}}{\left(r_{1}^{2}\right)^{\alpha}\left(r_{2}^{2}\right)^{1-\beta}} \\
& \cdot F\left[\alpha, 1-\beta ; 1+\alpha-\beta ;\left(1-\frac{r^{2}}{r_{1}^{2}}\right)\left(1-\frac{r^{2}}{r_{2}^{2}}\right)\right],
\end{aligned}
$$

where $\alpha$ and $\beta$ are real numbers and $0<2 \alpha, 2 \beta<1$, while $r, r_{1}$ and $r_{2}$ are expressions defined in (1.3). 
Proof. It follows from (3.16) that

$$
\begin{aligned}
q_{3}\left(x, y ; x_{0}, y_{0}\right)= & k_{3} y^{1-2 \beta} y_{0}^{1-2 \beta}\left(r_{1}^{2}\right)^{-\alpha}\left(r_{2}^{2}\right)^{\beta-1} \\
& \cdot \sum_{i=0}^{\infty} \frac{(1+\alpha-\beta)_{i}(\alpha)_{i}(1-\beta)_{i}}{(2 \alpha)_{i}(2-2 \beta)_{i} i !}\left(1-\frac{r^{2}}{r_{1}^{2}}\right)^{i}\left(1-\frac{r^{2}}{r_{2}^{2}}\right)^{i} \\
& \cdot F\left(\alpha+\beta-1, \alpha+i ; 2 \alpha+i ; 1-\frac{r^{2}}{r_{1}^{2}}\right) \\
& \cdot F\left(1-\alpha-\beta, 1-\beta+i ; 2-2 \beta+i ; 1-\frac{r^{2}}{r_{2}^{2}}\right),
\end{aligned}
$$

Then in view of the inequalities

$$
F\left(\alpha+\beta-1, \alpha+i ; 2-2 \alpha+i ; 1-\frac{r^{2}}{r_{1}^{2}}\right) \leqslant \frac{(2 \alpha)_{i} \Gamma(2 \alpha) \Gamma(1-\beta)}{(1+\alpha-\beta){ }_{i} \Gamma(1+\alpha-\beta) \Gamma(\alpha)}
$$

and

$$
F\left(1-\alpha-\beta, 1-\beta+i ; 2-2 \beta+i ; 1-\frac{r^{2}}{r_{2}^{2}}\right) \leqslant \frac{(2-2 \beta)_{i} \Gamma(2-2 \beta) \Gamma(\alpha)}{(1+\alpha-\beta){ }_{i} \Gamma(1+\alpha-\beta) \Gamma(1-\beta)},
$$

by (3.33) we arrive at inequality (3.32).

By the known formula [6]

$$
\begin{aligned}
F(a, b ; a & +b ; z)=-\frac{\Gamma(a+b)}{\Gamma(a) \Gamma(b)} F(a, b ; 1 ; 1-z) \ln (1-z) \\
& +\frac{\Gamma(a+b)}{\Gamma^{2}(a) \Gamma^{2}(b)} \sum_{j=0}^{\infty} \frac{\Gamma(a+j) \Gamma(b+j)}{(j !)^{2}}[2 \psi(1+j)-\psi(a+j)-\psi(b+j)](1-z)^{j}
\end{aligned}
$$

$(-\pi<\arg (1-z)<\pi, a, b \neq 0,-1,-2, \ldots)$, it follows from (3.32) that [4] the function $q_{3}\left(x, y ; x_{0}, y_{0}\right)$ has a logarithmic singularity at $r=0$.

Lemma 4. If the curve $\Gamma$ satisfies the aforementioned conditions, then

$$
\int_{\Gamma} x^{2 \alpha} y^{2 \beta}\left|\frac{\partial q_{3}\left(x, y ; x_{0}, y_{0}\right)}{\partial n}\right| d s \leqslant C_{1},
$$

where $C_{1}$ is a constant.

Proof. Inequality (3.34) follows conditions (3.1) and formula (3.10).

Formulae (3.3) show that as $\mu_{3}(s) \equiv 1$, the double layer potential has a discontinuity as the point $(x, y)$ passes through the curve $\Gamma$. For an arbitrary continuous density $\mu_{3}(s)$ the following theorem holds.

Theorem 1. The double layer potential $w^{(3)}\left(x_{0}, y_{0}\right)$ has limits as the point $\left(x_{0}, y_{0}\right)$ tends to a point $(x(s), y(s))$ at the curve $\Gamma$ from inside or outside. We denote limit of the values $w_{i}^{(3)}\left(x_{0}, y_{0}\right)$ from inside by $w^{(3)}(s)$, while the limit from outside is $w_{e}^{(3)}(s)$, and the formulae hold:

$$
w_{i}^{(3)}(t)=-\frac{1}{2} \mu_{3}(t)+\int_{0}^{l} \mu_{3}(s) K_{3}(s, t) d s
$$

and

$$
w_{e}^{(3)}(t)=\frac{1}{2} \mu_{3}(t)+\int_{0}^{l} \mu_{3}(s) K_{3}(s, t) d s
$$


where

$$
K_{3}(s, t)=[x(s)]^{2 \alpha}[y(s)]^{2 \beta} \frac{\partial}{\partial n}\left\{q_{3}\left[x(s), y(s) ; x_{0}(t), y_{0}(t)\right]\right\} .
$$

Proof. The theorem follows Lemmata 1-4.

The function

$$
w_{0}^{(3)}(s)=\int_{0}^{l} \mu_{3}(t) K_{3}(s, t) d t
$$

is continuous as $0 \leqslant s \leqslant l$ that is implied by the proof of Theorem 1 . By Theorem 1 and the continuity of the functions $w_{0}^{3}(s)$ and $\mu_{3}(s)$ as $0 \leqslant s \leqslant l$, we conclude that the double layer potential $w^{(3)}\left(x_{0}, y_{0}\right)$ is a continuous function inside the domain $\Omega$ up to the curve $\Gamma$. In the same way, $w^{(3)}\left(x_{0}, y_{0}\right)$ is continuous outside the domain $D$ up to the curve $\Gamma$.

In conclusion we observe that the results obtained in the present paper play important role while solving boundary value problems for the equation $H_{\alpha, \beta}^{0}(u)=0$. At that, a solution of the problem is sought as third double layer potential (3.2) with an unknown density $\mu_{3}(s)$ and to find this density, one can employ the known theorem of Fredholm equations of second kind.

\section{BIBLIOGRAPHY}

1. C. Miranda. Equazioni alle derivate parziali di tipo ellittico. Springer, Berlin (1955). (in Italian).

2. N.M. Günter. Potential theory and its application to main problems in mathematical physics. Gostekhizdat, Moscow (1953). (in Russian).

3. R.P. Gilbert. Function theoretic methods in partial differential equations. mathematics in science and engineering. Academic Press, New York (1969).

4. A. Hasanov. Fundamental solutions of generalized bi-axially symmetric Helmholtz equation // Compl. Variab. Ellip. Equat. 52:8, 673-683 (2007).

5. P. Appell, J. Kampé de Fériet. Fonctions hypergéométriques et hypersphériques: polynômes d'Hermite. Gauthier-Villars. Paris (1926).

6. A. Erdélyi, W. Magnus, F. Oberhettinger, G. Tricomi. Higher transcendental functions. I. McGrawHill Book Co., New York (1953).

7. H.M. Srivastava, P.W. Karlsson. Multiple Gaussian hypergeometric series. Halsted Press (Ellis Horwood Limited, Chicherster), John Wiley and Sons. New York (1985).

8. R.M. Mavlyaviev. Construction of fundamental solutions to B-elliptic equations with minor terms // Russ. Math. 61:6, 60-65 (2017).

9. M.M. Smirnov. Degenerate hyperbolic equations. Nauka, Moscow (1966). (in Russian).

10. S.P. Pul'kin. Some boundary value problems for equation $u_{x x} \pm u_{y y}+\frac{p}{x} u_{x}=0 / /$ Bull. Kuibyshev Pedag. Inst. 21, 3-54 (1958). (in Russian).

11. H.M. Srivastava, A. Hasanov, J. Choi. Double-layer potentials for a generalized bi-axially symmetric Helmholtz equation // Sohag J. Math. 2:1, 1-10 (2015).

12. M. Rassias, A. Hasanov. Fundamental solutions of two degenerated elliptic equations and solutions of boundary value problems in infinite area // Int. J. Appl. Math. Stat. 8:M07, 87-95 (2007).

13. J.L. Burchnall, T.W. Chaundy. Expansions of Appell's double hypergeometric functions // Quart. J. Math. Oxford Ser. 11:1, 249-270 (1940).

14. I.S. Gradshtejn, I.M. Ryzhik. Tables of series, products, and integrals. Fizmatgiz, Moscow (1962). [V. 1,2. Verlag Harri Deutsch, Frankfurt-am-Main (1981).]

Tukhtasin Gulamzhanovich Ergashev,

Tashkent Institute of Irrigation

and Agricultural Mechanization Engineers,

Kari-Niyaz str. 39,

100000, Tashkent, Uzbekistan

E-mail: ertuhtasin@mail.ru 